\title{
Investigation of the Use of Stereo-Pair Data Sets in Electron Tomography Characterization of Organic-Based Solar Cells
}

\author{
Jessica A. Alexander ${ }^{1}$, Michael F. Durstock ${ }^{2}$, Christopher E. Tabor ${ }^{2}$, Benjamin J. Leever ${ }^{2}$, Lawrence F. \\ Drummy $^{2}$, Michael D. Clark ${ }^{2,3}$, Dennis P. Butcher ${ }^{2,3}$, Frank J. Scheltens ${ }^{1}$ and David W. McComb ${ }^{1}$ \\ 1. Department of Materials Science and Engineering, The Ohio State University, College Road, \\ Columbus OH 43210, United States \\ 2. Materials and Manufacturing Directorate, Air Force Research Laboratory, WPAFB, Ohio 45433, \\ United States \\ 3. UES, Inc., Dayton, Ohio 45432, United States
}

The processes that generate current in organic photovoltaics (OPVs) are highly dependent on the micro-and nano-structure of the devices, especially at the donor-acceptor (D-A) interface. Light trapping strategies have been proposed to tailor absorption of incident sunlight and generate more photocurrent at the D-A interface. Recent studies have reported the use of a range of plasmonic nanostructures, such as nanoparticles, slit arrays and nanohole arrays to improve the power conversion efficiency (PCE) in OPVs $[1,2]$. While incorporation of plasmonic nanostructures for light trapping in thin-film PV cells is an attractive solution for enhancement of the optical absorption and current density in an OPV without increasing the thickness of its active layers, little is known about the detailed structure, chemistry and bonding between the active layer and the plasmonic nanostructures. The understanding of this interface is vital to understanding why these nanoparticles improve the efficiency of such devices. This knowledge will provide a foundation for the engineering of new OPV devices with improved PCE.

OPVs have been fabricated that consist an active layer comprised of a blend of poly( 3 hexylthiophene) (P3HT) and [6,6]phenyl $\mathrm{C}_{61}$ butyric acid methylester (PCBM). It is within this layer that nanoparticles for the plasmonic enhancement are incorporated. High-resolution transmission electron microscopy (HRTEM) may be utilized to obtain 2-D projections of the films and plasmonic nanoparticles, which may then be used to investigate the structure of the interfaces and characterize the quality of the devices fabricated. However, the drawback of HRTEM is that the image is a projection of the sample in 2-D. Thus, it is difficult to characterize the interactions that are occurring between the acceptor and donor domains within the polymer films because the 2-D image does not provide any information about the interfaces within the volume of the sample. Furthermore, the metal nanoparticles are represented in a 2D projection and information of their 3-D arrangement within the active layer is difficult to discern. Therefore, methodologies to obtain 3-D representations of the films are essential. Electron tomography (ET) is one such technique.

Unfortunately, when ET data collection is attempted on the OPV samples, electron beam damage has been observed. The electron beam damage is extensive, causing the sample to completely degrade before the entire data set has been collected. The challenge is to collect "enough" data to reconstruct the 3-D representation of the sample before any significant damage occurs, otherwise the structure of the sample changes, rendering any subsequent collected data useless. One possibility to overcoming this problem is to collect a stereo-pair data set. In stereo-pair imaging, 2-D images are used to create the perception of depth (see Figure 1). Thus, the idea behind applying this to ET is that images may be collected and combined so that the 3-D depth may be inferred from the images. This is a viable option because it requires collecting many fewer images of the sample. Thus, it is possible that the entire 
stereo-pair data set may be collected before the sample degrades since collecting fewer images corresponds to less time that the sample is under the electron beam. The relevant question, is "does a stereo-pair data set have enough information to identify characteristic differences between the films with and without the nanoparticles". In this work we are exploring the answer to this question by applying the stereo-data set method to P3HT-PCBM devices to determine if it is even possible to collect he complete stereo-pair data set before the sample deteriorates, and if it does, is there sufficient information to answer questions about the D-A interface and the role of the nanoparticles.

In addition to overcoming the sample damage issue, the diffraction effects of the OPV devices must be determined since ET will not work for samples that have high scattering. This is because ET requires that the signal measured is a projection of the sample, which is not the case for high scattering samples. However, STEM-HAADF tomography could be utilized if the scattering of the OPVs is too large, since electrons that have scattered to high angles, rather than diffracted, are used to form the image.

Our goal is to extract a 3-D representation of the morphological features in the OPV films, including the morphology of the interfaces between the active layer and the nanoparticles. This reconstruction can then be compared to the reconstructions of devices that do not contain. Ultimately, this will improve our understanding of how the nanoparticles affect the efficiency of the devices.

\section{$\underline{\text { References }}$}

[1] H. A. Atwater, A. Polman, Nature Materials 9 (2010), p. 205.

[2] V.E. Ferry, J.N. Munday, H.A. Atwater, Advanced Materials 22 (2010), p. 4794.

[3] The authors acknowledge the Air Force Office of Scientific Research and the Air Force Research Laboratory Matierals and Manufacturing Directorate for funding as well as The Ohio State University Center for Electron Microscopy and Analysis for technical support.
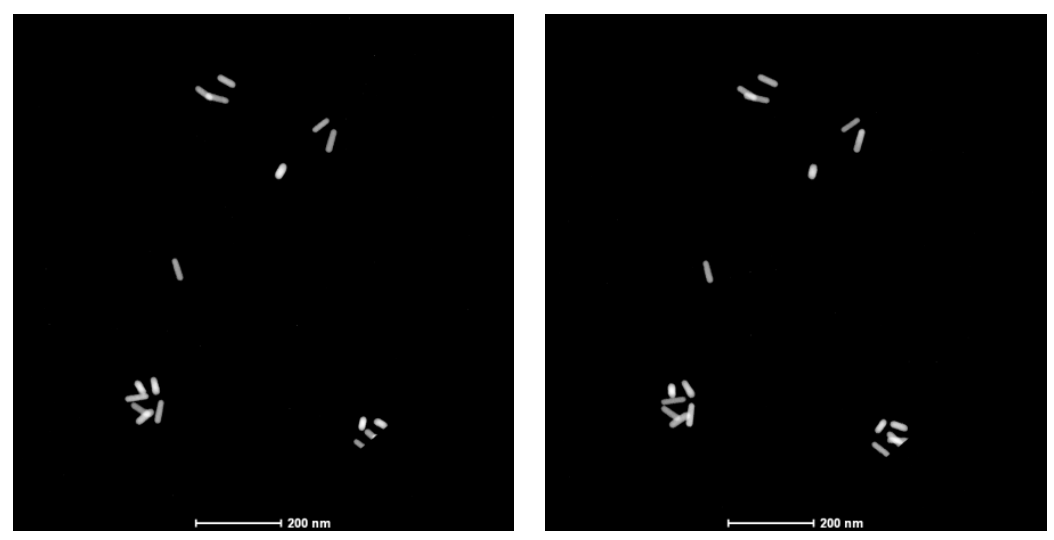

Figure 1. Stereo pair of gold nano rod clusters in P3HT:PCBM BHJ OPV active layer. 